\title{
Sharp threshold functions for random intersection graphs via a coupling method.
}

\author{
Katarzyna Rybarczyk \\ Faculty of Mathematics and Computer Science, \\ Adam Mickiewicz University, 60-769 Poznań, Poland \\ kryba@amu.edu.pl \\ Submitted: Nov 25, 2009; Accepted: Feb 7, 2011; Published: Feb 14, 2011 \\ Mathematics Subject Classification: 05C80 \\ keywords: random intersection graphs, threshold functions, \\ connectivity, Hamilton cycle, perfect matching, coupling
}

\begin{abstract}
We present a new method which enables us to find threshold functions for many properties in random intersection graphs. This method is used to establish sharp threshold functions in random intersection graphs for $k$-connectivity, perfect matching containment and Hamilton cycle containment.
\end{abstract}

\section{Introduction}

In a general random intersection graph $\overline{\mathcal{G}}\left(n, m, \mathcal{P}_{(m)}\right)$, as defined in [9], each vertex $v$ from a vertex set $\mathcal{V}(|\mathcal{V}|=n)$ is assigned independently a subset of features $W_{v} \subseteq \mathcal{W}$ from an auxiliary set of features $\mathcal{W}(|\mathcal{W}|=m)$. Namely, for any vertex $v \in \mathcal{V}$, independently of all other vertices, first a cardinality of $W_{v}$ is chosen according to the probability distribution $\mathcal{P}_{(m)}=\left(P_{0}, \ldots, P_{m}\right)$, and then the set $W_{v}$ is picked uniformly at random from all subsets of $\mathcal{W}$ having the chosen cardinality. Two vertices $v$ and $v^{\prime}$ are adjacent in a general intersection graph $\overline{\mathcal{G}}\left(n, m, \mathcal{P}_{(m)}\right)$ if and only if $W_{v}$ and $W_{v^{\prime}}$ intersect. In this article we concentrate on the widely studied random intersection graph model $\mathcal{G}(n, m, p)$ first defined in $[11,17]$ which is a special case of the one above-mentioned. However the obtained results may be extended to a wider subclass of the $\overline{\mathcal{G}}\left(n, m, \mathcal{P}_{(m)}\right)$ model, which will be also discussed. In $\mathcal{G}(n, m, p)$, as defined in $[11,17]$, the cardinality of $W_{v}$ has the binomial distribution $\operatorname{Bin}(m, p)$, i.e. $\operatorname{Pr}\left\{w \in W_{v}\right\}=p$ independently for all $v \in \mathcal{V}$ and $w \in \mathcal{W}$. Usually, it is assumed that $m=n^{\alpha}$ for some constant $\alpha>0$ (see for example $[2,6,8,11,16,17,18])$. However the main theorem of this article does not require this additional assumption. 
Obviously, $\operatorname{Pr}\left\{\left\{v, v^{\prime}\right\} \in E(\mathcal{G}(n, m, p))\right\}=1-\left(1-p^{2}\right)^{m}$ for any distinct $v, v^{\prime} \in \mathcal{V}$. Therefore one could expect that there is some relation between $\mathcal{G}(n, m, p)$ and a random graph $G(n, \hat{p})$ with edges appearing independently with probability $\hat{p}$ for $\hat{p}$ approximately $1-\left(1-p^{2}\right)^{m}$. It follows from the results on subgraph containment as presented in $[11,16]$, in general, these are not equivalence relations since the structures of $\mathcal{G}(n, m, p)$ and $G(n, \hat{p})$ differ significantly. However it was shown in [8] that for large $m$ (i.e. $m=n^{\alpha}$ and $\alpha>6)$, dependencies between edge appearances in $\mathcal{G}(n, m, p)$ are small and the models have asymptotically the same properties. The equivalence theorem is extended to $m=n^{\alpha}$ and $\alpha \geq 3$ (see [15]), but for $m=n^{\alpha}$ and $\alpha<3$ it is not true in general (see for example $[11,16]$ ). In the context of the results stated above it seems intriguing that for $m=n^{\alpha}$ and $\alpha>1$ the threshold functions of connectivity and phase transition in $\mathcal{G}(n, m, p)$ and $G(n, \hat{p})$ coincide (see $[2,7,17]$ ) even though the models differ a lot (for example the expected number of triangles in $\mathcal{G}(n, m, p)$ significantly exceeds the expected number of triangles in $G(n, \hat{p})$ for $\alpha<3)$. One of the aims of this paper is to get an improved understanding of the phenomena by a closer insight into the structure of $\mathcal{G}(n, m, p)$ and to use this knowledge to establish sharp threshold functions for other important properties of $\mathcal{G}(n, m, p)$.

Our work is partially inspired by the result of Efthymiou and Spirakis [6]. However the method significantly differs from the one used in [6] and therefore it enables us to obtain sharper threshold functions for the property of Hamilton cycle containment than those from [6].

The article is organised as follows. In Section 2 we present and prove the main theorem which relates $\mathcal{G}(n, m, p)$ to $G(n, \hat{p})$. In Section 3 the theorem is used to study properties of $\mathcal{G}(n, m, p)$. In particular, an alternative short proof of the connectivity theorem shown in [17] is given. Moreover, results concerning sharp threshold functions for Hamilton cycle containment, perfect matching containment and $k$-connectivity are proved. The method introduced here is strong enough to give some partial results on the threshold functions for other properties of $\mathcal{G}(n, m, p)$. However we present here graph properties for which the threshold functions obtained by our method are tight at least for $m=n^{\alpha}$ and $\alpha>1$. In Section 4 extensions of the results to a wider subclass of the general random intersection graph model are presented. Moreover some interesting questions related to the main theorem are discussed.

All limits in the paper are taken as $n \rightarrow \infty$. Throughout the paper we use the notation $a_{n}=o\left(b_{n}\right)$ if $a_{n} / b_{n} \rightarrow 0$ and $a_{n} \sim b_{n}$ if $a_{n} / b_{n} \rightarrow 1$. Also by $\operatorname{Bin}(n, p)$ and $\operatorname{Po}(\lambda)$ we denote the binomial distribution with parameters $n, p$ and the Poisson distribution with expected value $\lambda$, respectively. Moreover if a random variable $X$ is stochastically dominated by $Y$ we write $X \prec Y$. We also use the phrase "with high probability" to say with probability tending to one as $n$ tends to infinity.

\section{Main Result}

Recall that for the family $\mathcal{G}$ of all graphs with a vertex set $\mathcal{V}$, we call $\mathcal{A} \subseteq \mathcal{G}$ an increasing property if $\mathcal{A}$ is closed under isomorphism and $G \in \mathcal{A}$ implies $G^{\prime} \in \mathcal{A}$ for all $G^{\prime} \in \mathcal{G}$ 
such that $E(G) \subseteq E\left(G^{\prime}\right)$. The theorem stated below relates $\mathcal{G}(n, m, p)$ to $G(n, \hat{p})$ for increasing properties. A motivation for the investigation in a comparison was the fact that, for $m=n^{\alpha}$ and $\alpha>1$, if $p$ and $\hat{p}$ are connectivity threshold functions of $\mathcal{G}(n, m, p)$ and $G(n, \hat{p})$, respectively, then $\operatorname{Pr}\left\{\left\{v, v^{\prime}\right\} \in E(\mathcal{G}(n, m, p))\right\} \sim 1-\left(1-p^{2}\right)^{m} \sim m p^{2} \sim \hat{p}$ (see [17]). In the proof of the theorem we explain that this is due to the fact that $n p \rightarrow 0$. Surprisingly, in some cases the comparison also gives tight results for $n p \nrightarrow 0$, however with $\hat{p}$ differing from $1-\left(1-p^{2}\right)^{m}$. This is due to the fact that as $n p \rightarrow \infty$ the number of large cliques in $\mathcal{G}(n, m, p)$ increases compared to $G(n, \hat{p})$ and thus both models have significantly different edge structures. Basically, as $n p \rightarrow \infty$ and $\hat{p}=(1+o(1)) m p / n$, $\mathcal{G}(n, m, p)$ has more edges than $G(n, \hat{p})$, however both models have the same number of isolated vertices. In the theorem we have the case $n m p \rightarrow \infty$ instead of $n p \rightarrow \infty$, since the thesis also holds true in this case. However as $n m p \rightarrow \infty$ and $n p \nrightarrow \infty$ the results obtained using the theorem will not be tight.

Theorem 1. Let $\mathcal{A}$ be an increasing property, $m p^{2}<1$, and

$$
\hat{p}_{-}= \begin{cases}m p^{2}\left(1-(n-2) p-\frac{m p^{2}}{2}\right) & \text { for } n p=o(1) ; \\ \frac{m p}{n}\left(1-\frac{\omega}{\sqrt{m n p}}-\frac{2}{n p}-\frac{m p}{2 n}\right) & \text { for } n m p \rightarrow \infty \\ & \text { and some } \omega \rightarrow \infty, \omega=o(\sqrt{m n p}) .\end{cases}
$$

If

$$
\operatorname{Pr}\left\{G\left(n, \hat{p}_{-}\right) \in \mathcal{A}\right\} \rightarrow 1
$$

then

$$
\operatorname{Pr}\{\mathcal{G}(n, m, p) \in \mathcal{A}\} \rightarrow 1 .
$$

The main ingredient of the proof is a comparison of $\mathcal{G}(n, m, p)$ and $G(n, \hat{p})$ using intermediate auxiliary graphs. The comparison is made by a sequence of couplings and measuring the distance between distributions of auxiliary graph valued random variables. First we introduce necessary definitions and notation.

Let $M$ be a random variable with range in the set of non-negative integers (in the simplest case $M$ is a given positive integer with probability one). By $\mathcal{G}_{*}(n, M)$ we denote a random graph with vertex set $\mathcal{V}$ and edge set constructed by sampling $M$ times with repetition elements from the set of all two element subsets of $\mathcal{V}$. A subset $\left\{v, v^{\prime}\right\}$ is an edge of $\mathcal{G}_{*}(n, M)$ if and only if it is sampled at least once. If $M$ equals a constant $t$ with probability one, has the binomial distribution, or the Poisson distribution, we write $\mathcal{G}_{*}(n, t), \mathcal{G}_{*}(n, \operatorname{Bin}(\cdot, \cdot))$, or $\mathcal{G}_{*}(n, \operatorname{Po}(\cdot))$, respectively.

For any random variables $G_{1}$ and $G_{2}$ with values in a countable set $A$, by the total variation distance we mean

$$
\begin{aligned}
d_{T V}\left(G_{1}, G_{2}\right) & =\max _{A^{\prime} \subseteq A}\left|\operatorname{Pr}\left\{G_{1} \in A^{\prime}\right\}-\operatorname{Pr}\left\{G_{2} \in A^{\prime}\right\}\right| \\
& =\frac{1}{2} \sum_{a \in A}\left|\operatorname{Pr}\left\{G_{1}=a\right\}-\operatorname{Pr}\left\{G_{2}=a\right\}\right| .
\end{aligned}
$$


By a coupling $\left(G_{1}, G_{2}\right)$ of two random variables $G_{1}$ and $G_{2}$ we mean a choice of a probability space on which a random vector $\left(G_{1}^{\prime}, G_{2}^{\prime}\right)$ is defined and $G_{1}^{\prime}$ and $G_{2}^{\prime}$ have the same distributions as $G_{1}$ and $G_{2}$, respectively. For simplicity of notation we will not differentiate between $\left(G_{1}^{\prime}, G_{2}^{\prime}\right)$ and $\left(G_{1}, G_{2}\right)$. For two graph valued random variables $G_{1}$ and $G_{2}$ we write

$$
G_{1} \preceq G_{2} \quad \text { or } \quad G_{1} \preceq_{1-o(1)} G_{2},
$$

if there exists a coupling $\left(G_{1}, G_{2}\right)$, such that under the coupling $G_{1}$ is a subgraph of $G_{2}$ with probability 1 or $1-o(1)$, respectively. Moreover, we write

$$
G_{1}=G_{2}
$$

if $G_{1}$ and $G_{2}$ have the same probability distribution (equivalently there exists a coupling $\left(G_{1}, G_{2}\right)$ such that $G_{1}=G_{2}$ with probability one).

It is simple to construct suitable couplings which implies the following fact.

Fact 1. (i) Let $M_{n}$ be a sequence of random variables and let $a_{n}$ be a sequence of numbers. If

$$
\operatorname{Pr}\left\{M_{n} \geq a_{n}\right\}=o(1) \quad\left(\operatorname{Pr}\left\{M_{n} \leq a_{n}\right\}=o(1)\right),
$$

then

$$
\mathcal{G}_{*}\left(n, M_{n}\right) \preceq_{1-o(1)} \mathcal{G}_{*}\left(n, a_{n}\right) \quad\left(\mathcal{G}_{*}\left(n, a_{n}\right) \preceq_{1-o(1)} \mathcal{G}_{*}\left(n, M_{n}\right)\right) .
$$

(ii) If a random variable $M$ is stochastically dominated by $M^{\prime}$ (i.e. $M \prec M^{\prime}$ ), then

$$
\mathcal{G}_{*}(n, M) \preceq \mathcal{G}_{*}\left(n, M^{\prime}\right) .
$$

The proof of the next fact is analogous to the proof of Fact 2 in [15].

Fact 2. Let $\left(G_{i}\right)_{i=1, \ldots, m}$ and $\left(G_{i}^{\prime}\right)_{i=1, \ldots, m}$ be sequences of independent random graphs. If

$$
G_{i} \preceq G_{i}^{\prime}, \text { for all } i=1, \ldots, m
$$

then

$$
\bigcup_{i=1}^{m} G_{i} \preceq \bigcup_{i=1}^{m} G_{i}^{\prime} .
$$

Proof of Theorem 1. Let $w \in \mathcal{W}$. Denote by $V_{w}$ the set of vertices which have chosen feature $w$ and put $X_{w}=\left|V_{w}\right|$. Let $\mathcal{G}\left[V_{w}\right]$ be a graph with vertex set $\mathcal{V}$ and edge set containing those edges which have both ends in $V_{w}$ (i.e. its edges form a clique with the vertex set $\left.V_{w}\right)$. We can construct a coupling $\left(\mathcal{G}_{*}\left(n,\left\lfloor X_{w} / 2\right\rfloor\right), \mathcal{G}\left[V_{w}\right]\right)$ which implies

$$
\mathcal{G}_{*}\left(n,\left\lfloor X_{w} / 2\right\rfloor\right) \preceq \mathcal{G}\left[V_{w}\right],
$$

in the following way. Given the value of $X_{w}$, first we generate an instance $G_{w}$ of $\mathcal{G}_{*}\left(n,\left\lfloor X_{w} / 2\right\rfloor\right)$. Let $Y_{w}$ be the number of non-isolated vertices in $G_{w}$. By definition $Y_{w}$ is at most $X_{w}$, therefore $V_{w}$ may be chosen to be a union of the set of non-isolated vertices in $G_{w}$ and $X_{w}-Y_{w}$ vertices chosen uniformly at random from the remaining ones. 
Graphs $\mathcal{G}_{*}\left(n,\left\lfloor X_{w} / 2\right\rfloor\right), w \in \mathcal{W}$, are independent, and $\mathcal{G}\left[V_{w}\right], w \in \mathcal{W}$, are independent. Thus by Fact 2 and the definition of $\mathcal{G}(n, m, p)$, we have

$$
\bigcup_{w \in \mathcal{W}} \mathcal{G}_{*}\left(n,\left\lfloor X_{w} / 2\right\rfloor\right) \preceq \bigcup_{w \in \mathcal{W}} \mathcal{G}\left[V_{w}\right]=\mathcal{G}(n, m, p) .
$$

Since $X_{w}, w \in \mathcal{W}$, are independent random variables and $\mathcal{G}\left[V_{w}\right], w \in \mathcal{W}$, are independent as well, by the above equation and the definition of $\mathcal{G}_{*}(n, \cdot)$,

$$
\mathcal{G}_{*}\left(n, \sum_{w \in \mathcal{W}}\left\lfloor X_{w} / 2\right\rfloor\right)=\bigcup_{w \in \mathcal{W}} \mathcal{G}_{*}\left(n,\left\lfloor X_{w} / 2\right\rfloor\right) \preceq \mathcal{G}(n, m, p)
$$

Now consider the following two cases

CASE 1: $n p=o(1)$.

Notice that

$$
\sum_{w \in \mathcal{W}} \mathbb{I}_{w} \prec \sum_{w \in \mathcal{W}}\left\lfloor X_{w} / 2\right\rfloor
$$

where

$$
\mathbb{I}_{w}= \begin{cases}1, & \text { if } X_{w} \geq 2 \\ 0, & \text { otherwise }\end{cases}
$$

The random variable $Z_{1}=\sum_{w \in \mathcal{W}} \mathbb{I}_{w}$ has the binomial distribution $\operatorname{Bin}(m, q)$, where $q=\operatorname{Pr}\left\{X_{w} \geq 2\right\}$, therefore by Fact 1 (ii),

$$
\mathcal{G}_{*}(n, \operatorname{Bin}(m, q)) \preceq \mathcal{G}_{*}\left(n, \sum_{w \in \mathcal{W}}\left\lfloor X_{w} / 2\right\rfloor\right) .
$$

Let $M_{1}$ and $M_{2}$ be random variables with the binomial distribution $\operatorname{Bin}(m, q)$ and the Poisson distribution $\operatorname{Po}(m q)$, respectively. A simple calculation shows that in $\mathcal{G}_{*}\left(n, M_{1}\right)$ each edge appears independently with probability $1-\exp \left(-m q /\left(\begin{array}{l}n \\ 2\end{array}\right)\right)($ see $[8])$. Therefore by properties of the total variation distance and the Poisson approximation of binomial random variables (see [8] and [1] or [15]), we have

$$
\begin{aligned}
d_{T V}\left(\mathcal{G}_{*}(n, \operatorname{Bin}(m, q)), G\left(n, 1-\exp \left(-m q /\left(\begin{array}{l}
n \\
2
\end{array}\right)\right)\right)\right) \\
\quad=d_{T V}\left(\mathcal{G}_{*}\left(n, M_{1}\right), \mathcal{G}_{*}\left(n, M_{2}\right)\right) \leq 2 d_{T V}\left(M_{1}, M_{2}\right) \leq 2 q \leq 2\left(\begin{array}{l}
n \\
2
\end{array}\right) p^{2}=o(1) .
\end{aligned}
$$

Moreover $q \geq \operatorname{Pr}\left\{X_{w}=2\right\}=\left(\begin{array}{l}n \\ 2\end{array}\right) p^{2}(1-p)^{n-2}$ and $1-\exp (-x) \geq x-x^{2} / 2$ for $x<1$ (recall that $m p^{2}<1$ by the assumptions of the theorem), thus

$$
p_{-}=m p^{2}\left(1-(n-2) p-\frac{m p^{2}}{2}\right) \leq 1-\exp \left(-m q /\left(\begin{array}{c}
n \\
2
\end{array}\right)\right) .
$$

Therefore by a standard coupling of $G(n, \cdot)$ we obtain

$$
G\left(n, p_{-}\right) \preceq G\left(n, 1-\exp \left(-m q /\left(\begin{array}{l}
n \\
2
\end{array}\right)\right)\right) .
$$


CASE 2: $n m p \rightarrow \infty$.

Notice that

$$
\frac{Z_{2}}{2}-m \prec \sum_{w \in \mathcal{W}}\left\lfloor X_{w} / 2\right\rfloor
$$

where $Z_{2}=\sum_{w \in \mathcal{W}} X_{w}$ has the binomial distribution Bin $(n m, p)$. By Fact 1(ii),

$$
\mathcal{G}_{*}\left(n, \frac{Z_{2}}{2}-m\right) \preceq \mathcal{G}_{*}\left(n, \sum_{w \in \mathcal{W}}\left\lfloor X_{w} / 2\right\rfloor\right) .
$$

By Chernoff's bound for the Poisson distribution (see [14] Lemma 1.2) for any function $\omega \rightarrow \infty, \omega=o(\sqrt{n m p})$,

$$
\operatorname{Pr}\left\{\frac{Z_{2}}{2}-m \leq \frac{n m p}{2}\left(1-\frac{\omega}{2 \sqrt{n m p}}-\frac{2}{n p}\right)\right\}=\operatorname{Pr}\left\{Z_{2} \leq n m p-\frac{\omega \sqrt{m n p}}{2}\right\}=o(1) .
$$

Moreover, the same bound applied to a random variable $Z_{3}$ with the Poisson distribution Po $\left(\frac{n m p}{2}\left(1-\frac{\omega}{\sqrt{n m p}}-\frac{2}{n p}\right)\right)$ gives

$$
\operatorname{Pr}\left\{Z_{3} \geq \frac{n m p}{2}\left(1-\frac{\omega}{2 \sqrt{n m p}}-\frac{2}{n p}\right)\right\}=\operatorname{Pr}\left\{Z_{3} \geq \mathbb{E} Z_{3}+\frac{\omega \sqrt{n m p}}{4}\right\}=o(1) .
$$

Therefore, using twice Fact 1(i), we get

$$
\mathcal{G}_{*}\left(n, \operatorname{Po}\left(\frac{n m p}{2}\left(1-\frac{\omega}{\sqrt{n m p}}-\frac{2}{n p}\right)\right)\right) \preceq_{1-o(1)} \mathcal{G}_{*}\left(n, \frac{Z_{2}}{2}-m\right) .
$$

Recall that, for any $\lambda$, in $\mathcal{G}_{*}(n, \operatorname{Po}(\lambda))$ each edge appears independently with probability $1-\exp \left(-\lambda /\left(\begin{array}{l}n \\ 2\end{array}\right)\right)$ (see [8]). Therefore

$$
G\left(n, 1-\exp \left(-\frac{m p}{n-1}\left(1-\frac{\omega}{\sqrt{n m p}}-\frac{2}{n p}\right)\right)\right)=\mathcal{G}_{*}\left(n, \operatorname{Po}\left(\frac{n m p}{2}\left(1-\frac{\omega}{\sqrt{n m p}}-\frac{2}{n p}\right)\right)\right) .
$$

Since

$$
\frac{m p}{n}\left(1-\frac{\omega}{\sqrt{n m p}}-\frac{2}{n p}-\frac{m p}{2 n}\right) \leq 1-\exp \left(-\frac{m p}{n-1}\left(1-\frac{\omega}{\sqrt{n m p}}-\frac{2}{n p}\right)\right),
$$

a standard coupling of $G(n, \cdot)$ implies

$$
G\left(n, p_{-}\right) \preceq G\left(n, 1-\exp \left(-\frac{m p}{n-1}\left(1-\frac{\omega}{\sqrt{n m p}}-\frac{2}{n p}\right)\right)\right) .
$$

In equations (3)-(10) we have established relations between $G\left(n, p_{-}\right)$and $\mathcal{G}(n, m, p)$ using intermediate auxiliary random graphs. From them we can deduce the assertion of the theorem.

First recall (see for example [8]) that if for some graph valued random variables $G_{1}$ and $G_{2}$

$$
d_{T V}\left(G_{1}, G_{2}\right)=o(1)
$$


then for any $a \in[0 ; 1]$ and any graph property $\mathcal{A}$

$$
\operatorname{Pr}\left\{G_{1} \in \mathcal{A}\right\} \rightarrow a \quad \text { iff } \quad \operatorname{Pr}\left\{G_{2} \in \mathcal{A}\right\} \rightarrow a .
$$

Now let $G_{1}$ and $G_{2}$ be two random graphs such that

$$
G_{1} \preceq G_{2} \quad \text { or } \quad G_{1} \preceq_{1-o(1)} G_{2} .
$$

Assume that for an increasing property $\mathcal{A}$,

$$
\operatorname{Pr}\left\{G_{1} \in \mathcal{A}\right\} \rightarrow 1 .
$$

Under the coupling $\left(G_{1}, G_{2}\right)$ given by (11) define event $\mathcal{H}:=\left\{G_{1} \subseteq G_{2}\right\}$. Then

$$
\begin{aligned}
1 \geq \operatorname{Pr}\left\{G_{2} \in \mathcal{A}\right\} & \geq \operatorname{Pr}\left\{G_{2} \in \mathcal{A} \mid \mathcal{H}\right\} \operatorname{Pr}\{\mathcal{H}\} \\
& \geq \operatorname{Pr}\left\{G_{1} \in \mathcal{A} \mid \mathcal{H}\right\} \operatorname{Pr}\{\mathcal{H}\} \\
& =\operatorname{Pr}\left\{\left\{G_{1} \in \mathcal{A}\right\} \cap \mathcal{H}\right\} \\
& =\operatorname{Pr}\left\{G_{1} \in \mathcal{A}\right\}+\operatorname{Pr}\{\mathcal{H}\}-\operatorname{Pr}\left\{\left\{G_{1} \in \mathcal{A}\right\} \cup \mathcal{H}\right\} \\
& \geq \operatorname{Pr}\left\{G_{1} \in \mathcal{A}\right\}+\operatorname{Pr}\{\mathcal{H}\}-1 \\
& =1+o(1)
\end{aligned}
$$

which means that

$$
\operatorname{Pr}\left\{G_{2} \in \mathcal{A}\right\} \rightarrow 1
$$

Therefore the above facts concerning the total variation distance and the properties of couplings combined with equations (3), (4), (5) and (6) imply Theorem 1 in the case $n p=o(1)$ and combined with equations (3), (7), (8), (9) and (10) imply the theorem in the case $n m p \rightarrow \infty$

\section{Sharp threshold functions}

Many graph properties in $G(n, \hat{p})$ follow the so called "minimum degree phenomenon". This means that with high probability the properties hold in $G(n, \hat{p})$ as soon as their necessary minimum degree condition is satisfied. In this section, using Theorem 1 , we show that the "minimum degree phenomenon" also holds in the case of $\mathcal{G}(n, m, p)$ for $m=n^{\alpha}$ and $\alpha>1$ and, to some extent, for $m=n^{\alpha}$ and $\alpha \leq 1$. Recall that while studying properties of $\mathcal{G}(n, m, p)$, it is standard to assume $m=n^{\alpha}$, and in this section we follow this convention. The properties considered are: $k$-connectivity, perfect matching containment and Hamilton cycle containment. All these properties are increasing and thus Theorem 1 may be used. Note that for $p_{k}$ considered in the theorems if $\alpha>1$ then $n p \rightarrow 0$ and if $\alpha \leq 1$, then $n p \rightarrow \infty$. The following theorems are proved.

Theorem 2. Let $m=n^{\alpha}$ and

$$
p_{1}= \begin{cases}\frac{\ln n+\omega}{m}, & \text { for } \alpha \leq 1 \\ \sqrt{\frac{\ln n+\omega}{n m}}, & \text { for } \alpha>1\end{cases}
$$


(i) If $\omega \rightarrow-\infty$, then with high probability $\mathcal{G}\left(n, m, p_{1}\right)$ is disconnected and does not contain a perfect matching.

(ii) If $\omega \rightarrow \infty$, then with high probability $\mathcal{G}\left(n, m, p_{1}\right)$ is connected and contains a perfect matching.

Theorems 3 and 4 consider the same properties. However they are stated separately since in the case $\alpha>1$ (Theorem 3) the obtained threshold functions are tight and for $\alpha \leq 1$ (Theorem 4) they may possibly be tightened by other methods.

Theorem 3. Let $k \geq 1$ be a constant integer, $\alpha>1, m=n^{\alpha}$ and

$$
p_{k}=\sqrt{\frac{\ln n+(k-1) \ln \ln n+\omega}{m n}} .
$$

1. (i) If $\omega \rightarrow-\infty$, then with high probability $\mathcal{G}\left(n, m, p_{k}\right)$ is not $k$-connected.

(ii) If $\omega \rightarrow \infty$, then with high probability $\mathcal{G}\left(n, m, p_{k}\right)$ is $k$-connected.

2. (i) If $\omega \rightarrow-\infty$, then with high probability $\mathcal{G}\left(n, m, p_{2}\right)$ does not contain a Hamilton cycle.

(ii) If $\omega \rightarrow \infty$, then with high probability $\mathcal{G}\left(n, m, p_{2}\right)$ contains a Hamilton cycle.

Theorem 4. Let $k \geq 1$ be a constant integer, $\alpha \leq 1, m=n^{\alpha}$,

$$
p_{k}=\frac{\ln n+(k-1) \ln \ln n+\omega}{m} .
$$

1. (i) If $\omega \rightarrow-\infty$, then with high probability $\mathcal{G}\left(n, m, p_{1}\right)$ is not $k$-connected.

(ii) If $\omega \rightarrow \infty$, then with high probability $\mathcal{G}\left(n, m, p_{k}\right)$ is $k$-connected.

2. (i) If $\omega \rightarrow-\infty$, then with high probability $\mathcal{G}\left(n, m, p_{1}\right)$ does not contain a Hamilton cycle.

(ii) If $\omega \rightarrow \infty$, then with high probability $\mathcal{G}\left(n, m, p_{2}\right)$ contains a Hamilton cycle.

Theorem 2 in its part concerning connectivity was obtained in [17]. However we state it here since it gives a global overview of the new method's implications and we are able to provide a new elegant proof of it. To the best of our knowledge the remaining results have not been proved before.

Proof of Theorems 2, 3 and 4. Denote

$$
\hat{p}_{k}=\frac{\ln n+(k-1) \ln \ln n+\omega}{n} .
$$

By some classical results (Erdős and Rényi [7], Bollobás and Thomason [5], Komlós and Szeméredi [12] and Bollobás [4]) 
1. (i) If $\omega \rightarrow-\infty$, then with high probability $G\left(n, \hat{p}_{1}\right)$ does not contain a perfect matching.

(ii) If $\omega \rightarrow \infty$, then with high probability $G\left(n, \hat{p}_{1}\right)$ contains a perfect matching.

2. (i) If $\omega \rightarrow-\infty$, then with high probability $G\left(n, \hat{p}_{k}\right)$ is not $k$-connected.

(ii) If $\omega \rightarrow \infty$, then with high probability $G\left(n, \hat{p}_{k}\right)$ is $k$-connected.

3. (i) If $\omega \rightarrow-\infty$, then with high probability $G\left(n, \hat{p}_{2}\right)$ does not contain a Hamilton cycle.

(ii) If $\omega \rightarrow \infty$, then with high probability $G\left(n, \hat{p}_{2}\right)$ contains a Hamilton cycle.

Since $k$-connectivity, Hamilton cycle containment and perfect matching containment are all increasing properties, parts (ii) of Theorems 2, 3 and 4 follow by Theorem 1 .

We are left with proving parts (i). The necessary condition for $k$-connectivity, perfect matching and Hamilton cycle containment are minimum degree at least $k, 1$ and 2, respectively. Therefore the following two lemmas imply parts (i) of the theorems.

Denote by $\delta(\mathcal{G}(n, m, p))$ the minimum degree of $\mathcal{G}(n, m, p)$.

Lemma 1. Let $k \geq 1$ be a constant integer, $\alpha>1$ and

$$
p_{k}=\sqrt{\frac{\ln n+(k-1) \ln \ln n+\omega}{n m}},
$$

(i) If $\omega \rightarrow-\infty$ then with high probability $\delta\left(\mathcal{G}\left(n, m, p_{k}\right)\right)<k$

(ii) If $\omega \rightarrow \infty$ then with high probability $\delta\left(\mathcal{G}\left(n, m, p_{k}\right)\right) \geq k$

Lemma 2. Let $\alpha \leq 1$ and

$$
p_{1}=\frac{\ln n+\omega}{m} .
$$

(i) If $\omega \rightarrow-\infty$ then with high probability $\delta\left(\mathcal{G}\left(n, m, p_{1}\right)\right)=0$.

(ii) If $\omega \rightarrow \infty$ then with high probability $\delta\left(\mathcal{G}\left(n, m, p_{1}\right)\right) \geq 1$.

Lemma 2 was shown in [17]. Part (ii) of Lemma 1 is easily obtained by the first moment method (see for example [10]). Moreover, to prove the theorems, only part (i) is needed. Its proof is a standard application of the second moment method (see [10]) and we sketch it for completeness.

We assume that $\omega=o(\ln n)$. Since the property "minimum degree at least $k$ " is increasing, the result for larger $\omega$ follows by a simple coupling argument applied to $\mathcal{G}(n, m, \cdot)$. The vertex degree analysis becomes complex for $\alpha$ near 1 due to edge dependencies. Therefore, to simplify arguments, instead of a random variable representing the degree of a vertex $v \in \mathcal{V}$, we study the auxiliary random variable

$$
Z_{v}=\mid\left\{\left(v^{\prime}, w\right): v \neq v^{\prime} \in \mathcal{V}, w \in W_{v} \text { and } w \in W_{v^{\prime}}\right\} \mid
$$


Let

$$
\xi_{v}=\left\{\begin{array}{ll}
1, & \text { if } Z_{v}=k-1 ; \\
0, & \text { otherwise; }
\end{array} \quad \text { and } \quad \xi=\sum_{v \in \mathcal{V}} \xi_{v} .\right.
$$

Clearly, if $\xi_{v}=1$, then the degree of the vertex $v$ is at most $k-1$. Therefore $\operatorname{Pr}\{\xi>0\} \rightarrow 1$ implies part (i) of Lemma 1.

Let $X_{v}=\left|W_{v}\right|$. By Chernoff's bound (see Theorem 2.1 in [10] or Lemma 1.1 in [14]),

$$
\operatorname{Pr}\left\{x_{-} \leq X_{v} \leq x_{+}\right\}=1-o\left(n^{-2}\right) \quad \text { for } x_{ \pm}=m p_{k}\left(1 \pm \sqrt{5 \ln n /\left(m p_{k}\right)}\right) .
$$

Moreover, given $X_{v}=x, Z_{v}$ has the binomial distribution $\operatorname{Bin}\left((n-1) x, p_{k}\right)$. Thus after careful calculation we get

$$
\begin{aligned}
\mathbb{E} \xi & =n \operatorname{Pr}\left\{Z_{v}=k-1\right\} \\
& =n \sum_{x=x_{-}}^{x_{+}} \operatorname{Pr}\left\{Z_{v}=k-1 \mid X_{v}=x\right\} \operatorname{Pr}\left\{X_{v}=x\right\}+o\left(n^{-2}\right) \\
& \geq \frac{1}{(k-1) !} \exp (-\omega+o(1))(1+o(1)) \rightarrow \infty .
\end{aligned}
$$

Let $v, v^{\prime} \in \mathcal{V}$ and $S=\left|W_{v} \cap W_{v^{\prime}}\right|$. Given $i \in\{0,1,2\}$ and $x, x^{\prime} \in\left[x_{-} ; x_{+}+2\right]$ denote by $\mathcal{H}\left(x, x^{\prime}, i\right)$ the event $\left\{X_{v}=x+i, X_{v^{\prime}}=x^{\prime}+i, S=i\right\}$. A calculation shows that if $i \in\{0,1,2\}$ and $x, x^{\prime} \in\left[x_{-} ; x_{+}+2\right]$, then uniformly over all $x, x^{\prime}$

$$
\begin{aligned}
\operatorname{Pr}\left\{\mathcal{H}\left(x, x^{\prime}, i\right)\right\} & =\operatorname{Pr}\left\{X_{v}=x+i\right\} \operatorname{Pr}\left\{X_{v^{\prime}}=x^{\prime}+i\right\} \operatorname{Pr}\left\{S=i \mid X_{v^{\prime}}=x^{\prime}+i, X_{v}=x+i\right\} \\
& =(1+o(1)) \operatorname{Pr}\left\{X_{v}=x\right\} \operatorname{Pr}\left\{X_{v^{\prime}}=x^{\prime}\right\} \operatorname{Pr}\{S=i\}
\end{aligned}
$$

Moreover, uniformly over all $x, x^{\prime} \in\left[x_{-} ; x_{+}+2\right]$, we have

$$
\begin{aligned}
\operatorname{Pr}\left\{Z_{v}=k-1, Z_{v^{\prime}}=\right. & \left.k-1 \mid \mathcal{H}\left(x, x^{\prime}, i\right)\right\} \\
& =(1+o(1)) \operatorname{Pr}\left\{Z_{v}=k-1 \mid X_{v}=x\right\} \operatorname{Pr}\left\{Z_{v^{\prime}}=k-1 \mid X_{v^{\prime}}=x\right\} .
\end{aligned}
$$

Denote $J=\left[x_{-}+2, x_{+}\right]$. Since $S$ has the binomial distribution $\operatorname{Bin}\left(m, p_{k}^{2}\right)$, and by Chernoff's bound applied to $X_{v}$ and $X_{v^{\prime}}$, we get

$\operatorname{Pr}\left\{X_{v} \notin J\right.$ or $X_{v^{\prime}} \notin J$ or $\left.S \notin\{0,1,2\}\right\}$

$$
\leq \operatorname{Pr}\left\{X_{v} \notin J\right\}+\operatorname{Pr}\left\{X_{v^{\prime}} \notin J\right\}+\operatorname{Pr}\{S \geq 3\}=o\left(n^{-2}\right) .
$$

Finally by the above calculation and (12) for $v \neq v^{\prime} \in \mathcal{V}$

$$
\begin{aligned}
\mathbb{E} \xi(\xi-1)= & n(n-1) \operatorname{Pr}\left\{Z_{v}=k-1, Z_{v^{\prime}}=k-1\right\} \\
\leq & n(n-1) \\
& \cdot \sum_{x=x_{-}}^{x_{+}} \sum_{x^{\prime}=x_{-}}^{x_{+}} \sum_{i=0}^{2} \operatorname{Pr}\left\{Z_{v}=k-1, Z_{v^{\prime}}=k-1 \mid \mathcal{H}\left(x, x^{\prime}, i\right)\right\} \operatorname{Pr}\left\{\mathcal{H}\left(x, x^{\prime}, i\right)\right\} \\
& +n(n-1) \operatorname{Pr}\left\{X_{v} \notin J \text { or } X_{v^{\prime}} \notin J \text { or } S \notin\{0,1,2\}\right\} \\
= & (1+o(1)) \operatorname{Pr}\left\{Z_{v}=k-1\right\} \operatorname{Pr}\left\{Z_{v^{\prime}}=k-1\right\}+o(1),
\end{aligned}
$$

which by the second moment method implies $\operatorname{Pr}\{\xi>0\} \rightarrow 1$. 


\section{Final remarks}

The obtained results may be extended to a wider class of the general random intersection graph model $\overline{\mathcal{G}}\left(n, m, \mathcal{P}_{(m)}\right)$. As an example we state here a uniform random intersection graph which is $\overline{\mathcal{G}}\left(n, m, \mathcal{P}_{(m)}\right)=\overline{\mathcal{G}}\left(n, m, \mathcal{P}_{d}\right)$ with probability distribution $\mathcal{P}_{(m)}=\mathcal{P}_{d}$ concentrated in $d=d(n)$, for some $d(n)$. More precisely in $\overline{\mathcal{G}}\left(n, m, \mathcal{P}_{d}\right)$, for all $v \in \mathcal{V}$, the set $W_{v}$ is chosen uniformly at random from all $d$-element subsets of $\mathcal{W}$. By Lemma 4 from [3] Theorems 2 and 3 hold true, if we assume that $\alpha>1$ and replace $p_{k}$ by $d_{k}=m p_{k}$ and $\mathcal{G}\left(n, m, p_{k}\right)$ by $\overline{\mathcal{G}}\left(n, m, \mathcal{P}_{d_{k}}\right)$.

As it clearly follows from Theorem 2, the couplings used in the proof of Theorem 1 are tight. However, in the case $n p \rightarrow \infty$ they do not always give the best results (see Theorem 4). Notice that in the case $\alpha<1$ it is easy to strengthen Lemma 2 by a simple application of Chernoff's bound.

Lemma 3. Let $\alpha<1$ and

$$
p_{1}=\frac{\ln n+\omega}{m} .
$$

If $\omega \rightarrow \infty$ then with high probability $\delta\left(\mathcal{G}\left(n, m, p_{1}\right)\right) \geq(1+o(1)) n \ln n / m$.

Therefore having in mind the "minimum degree phenomenon", we may conjecture that the threshold function given in Theorem 4 may be tightened. However we believe that to prove the following conjecture a new method has to be used.

Conjecture 1. Let $\alpha<1$,

$$
p=\frac{\ln n+\omega}{m},
$$

and $\omega \rightarrow \infty$. Then with high probability $\mathcal{G}(n, m, p)$ is $k$-connected for any constant $k$ and contains a Hamilton cycle.

This conjecture contains the assumption $\alpha<1$. Probably the case $\alpha=1$ is more complex. The thesis may be supported by the results concerning the degree distribution [18] and the phase transition [13] for $\alpha=1$. Although they consider $p$ near phase transition threshold, they show that, for some properties, there is a value of $\alpha$ for which an analysis of $\mathcal{G}(n, m, p)$ is complicated.

\section{Acknowledgements}

I would like to thank all colleagues attending our seminar and Mindaugas Bloznelis for their helpful remarks, which allowed me to improve the layout of the paper and remove some ambiguities. I am also grateful to the anonymous referee and my colleagues Erhard Godehardt and Michał Ren for making many helpful suggestions that improved the exposition. 


\section{References}

[1] Barbour, A. D., Holst, L. And Janson, S. Poisson Approximation. Oxford University Press, 1992

[2] Behrisch, M. Component evolution in random intersection graphs. Electron. J. Combin. 14, R17, 2007.

[3] Bloznelis, M., Jaworski, J. and Rybarczyk, K. Component evolution in a secure wireless sensor networks. Networks 53, 19-26, 2009.

[4] Bollobás, B. The evolution of sparse graphs, Graph Theory and Combinatorics (Cambridge 1983), 35-57, Academic Press, 1984.

[5] Bollobás, B. And Thomason, A. Random graphs of small order. Random Graphs '83 , Proceedings, Poznań, 1983, 47 - 97, 1985.

[6] Efthymiou, C. And Spirakis, P. G. On the existence of hamiltonian cycles in random intersection graphs. In: Automata, Languages and Programming 32nd International Colloquium, ICALP 2005, Lisbon, Portugal, 690-701, 2005.

[7] Erdős, P. And RÉnyi, A. On random graphs I. Publ. Math. Debrecen 6, 290-297, 1959.

[8] Fill, J. A., Scheinerman, E. R. And Singer-Cohen, K. B. Random intersection graphs when $m=\omega(n)$ : An equivalence theorem relating the evolution of the $G(n, m, p)$ and $G(n, p)$ models. Random Structures Algorithms 16, 156-176, 2000.

[9] Godehardt, E. AND Jaworski, J. Two models of random intersection graphs for classification. In: M. Schwaiger, O. Opitz, (eds.): Exploratory Data Analysis in Empirical Research, (Proceedings of the 25th Annual Conference of the Gesellschaft für Klassifikation e.V., University of Munich, 2001). Springer, 67-81, 2002.

[10] Janson, S., Łuczak, T. And Ruciński, A. Random Graphs. Wiley-Interscience, New York, 2000.

[11] Karoński, M., Scheinerman, E. R. And Singer-Cohen, K. B. On random intersection graphs: the subgraph problem. Combin. Probab. Comput. 8, 131-159, 1999.

[12] Komlós, J. And SzemÉREdi, E. Limit distributions for the existence of hamilton cycles in a random graph. Discrete Math. 43, 55 - 63, 1983.

[13] LagerÅs, A. N. And Lindholm, M. Electron. J. Combin. 15 (1), N10, 2008.

[14] Penrose, M. Random Geometric Graphs. Oxford University Press, 2003.

[15] RybarczyK, K. Equivalence of a random intersection graph and $G(n, p)$. Random Structures and Algorithms 38 (1-2), 205-234, 2011.

[16] RybarczyK, K. And Stark, D. Poisson approximation of the number of cliques in random intersection graphs. Journal of Applied Probability 47 (3), 826-840, 2010.

[17] Singer-Cohen, K. B. Random intersection graphs. PhD thesis, Department of Mathematical Sciences, The Johns Hopkins University, 1995.

[18] Stark, D. The vertex degree distribution of random intersection graphs. Random Structures and Algorithms 24, 249-258, 2004. 